\title{
Using vertical phase differences to better resolve 3D gravity wave structure
}

\author{
Corwin J. Wright ${ }^{1}$, Neil P. Hindley ${ }^{1}$, M. Joan Alexander ${ }^{2}$, Laura A. Holt ${ }^{2}$, and Lars Hoffmann ${ }^{3}$ \\ ${ }^{1}$ Centre for Space, Atmospheric and Oceanic Science, University of Bath, Bath, UK \\ ${ }^{2}$ Northwest Research Associates, Boulder, Colorado, USA \\ ${ }^{3}$ Jülich Supercomputing Centre, Forschungszentrum Jülich, Jülich, Germany
}

Correspondence: Corwin J. Wright (c.wright@bath.ac.uk)

Received: 1 April 2021 - Discussion started: 6 April 2021

Revised: 28 June 2021 - Accepted: 21 July 2021 - Published: 31 August 2021

\begin{abstract}
Atmospheric gravity waves (GWs) are a critically important dynamical mechanism in the terrestrial atmosphere, with significant effects on weather and climate. They are geographically ubiquitous in the middle and upper atmosphere, and thus, satellite observations are key to characterising their properties and spatial distribution. Nadir-viewing satellite instruments characterise the short horizontal wavelength portion of the GW spectrum, which is important for momentum transport; however, these nadir-sensing instruments have coarse vertical resolutions. This restricts our ability to characterise the 3D structure of these waves accurately, with important implications for our quantitative understanding of how these waves travel and how they drive the atmospheric circulation when they break. Here, we describe, implement and test a new spectral analysis method to address this problem. This method is optimised for the characterisation of waves in any three-dimensional data set where one dimension is of coarse resolution relative to variations in the wave field, a description which applies to GW-sensing nadirsounding satellite instruments but which is also applicable in other areas of science. We show that our new " $2 \mathrm{D}+1 \mathrm{ST}$ " method provides significant benefits relative to existing spectrally isotropic methods for characterising such waves. In particular, it is much more able to detect regional and height variations in observed vertical wavelength and able to properly characterise extremely vertically long waves that extend beyond the data volume.
\end{abstract}

\section{Introduction}

The Earth's atmosphere is extremely shallow in the vertical relative to the horizontal, with a depth of tens of kilometres for the well-mixed portion compared to a planetary circumference of $40000 \mathrm{~km}$. However, the strong effect of gravity causes atmospheric structure and dynamics to vary dramatically over this comparatively short vertical range. For example, the atmosphere has an $e$-folding scale height distance of only $7 \mathrm{~km}$, while gravity waves with vertical wavelengths 10 times that depth can transport significant momentum fluxes to the upper atmosphere (Liu et al., 2013; Ern et al., 2018).

This challenge becomes particularly acute when using stratospheric data from nadir-viewing infrared-sensing satellite instruments such as the Atmospheric Infrared Sounder (AIRS) on NASA's Aqua satellite and the Infrared Atmospheric Sounding Interferometer (IASI) instruments on the ESA's (European Space Agency) MetOp satellites (Aumann et al., 2003; Blumstein et al., 2004; Chahine et al., 2006; Hilton et al., 2012; Hoffmann et al., 2014). These instruments are optimised for measuring horizontal structures with scales of tens of kilometres or less but typically have vertical resolutions within the same order of magnitude, i.e. several kilometres or greater. Since the instruments typically have swath widths of over $1000 \mathrm{~km}$, while information on gravity waves is only useful in the stratosphere between altitudes $\sim 20$ $55 \mathrm{~km}$ (i.e. a depth of only $\sim 35 \mathrm{~km}$ ), the data only have a very small number of independent points per unit distance in the vertical relative to the number in the horizontal domain. 
Figure 1 illustrates this scale discrepancy using the example of a large-amplitude atmospheric gravity wave (GW) observed over the Southern Ocean in June 2010. Figure 1a shows the GW with the vertical axis stretched, as it would typically be shown in scientific publications and related contexts. Figure $1 \mathrm{~b}$ shows the same $\mathrm{GW}$ with its true aspect ratio, demonstrating that the complex vertical structure visible in Fig. 1a is entirely contained within an extremely narrow vertical layer close to the Earth's surface. As a result of these very different characteristic scales, it is technically challenging to study vertically varying atmospheric structures, such as GWs, using satellite remote sensing techniques.

This anisotropy causes major problems when using standard spectral analysis techniques, such as the Fourier transform and the Stockwell transform (S transform; Stockwell et al., 1996), to study the vertical variations associated with GWs in these data. The GWs observable by these instruments also have vertical scales greater than $10 \mathrm{~km}$ and horizontal scales of tens to hundreds of kilometres, but the relatively small number of measured points in the vertical domain relative to the horizontal, combined with the short vertical window range available in the data as imposed by the actual depth of the stratosphere and mesosphere, leads to severe limits on estimates of $\mathrm{GW}$ vertical wavelengths $\lambda_{z}$ when spectral analyses of this type are applied. An example of this issue is seen in Fig. 4e of Wright et al. (2017), where a 3D $\mathrm{S}$ transform method is applied to AIRS data but shown to only be capable of resolving two unique values of $\lambda_{z}$ in a volume where we would expect significant local $\lambda_{z}$ variations based on geophysical and measurement science grounds.

In this study, we address this problem by developing, implementing and testing a new spectral analysis method based on phase differences between two-dimensional $\mathrm{S}$ transforms of individual horizontal data levels. Our new method is able to characterise the vertical wavelengths of GWs measured in 3D nadir-sensed satellite data to a much higher degree of precision, with no significant reduction in data quality for any other analysed variables such as horizontal wavelength and wave amplitude. Although we only apply it here to the problem of GWs in nadir-sensed data, the technique is fully applicable to any spectral analysis problem involving multidimensional data sets where one of the dimensions has a significantly lower resolution than the others.

In Sect. 2, we describe the data we use for this study, and in Sect. 3 we outline our method, both conceptually and computationally, as we have implemented it. Section 4 then tests our method on simulated data, after which Sect. 5 applies it to three observational case studies. Finally, we summarise and discuss our results and draw conclusions in Sect. 6.

\section{Data}

We primarily use real temperature observations from the Atmospheric InfraRed Sounder (AIRS) on NASA's Aqua satel- lite, retrieved using the high-resolution retrieval method described by Hoffmann and Alexander (2009). These data have been extensively used for previous GW studies (e.g. Ern et al., 2017; Wright et al., 2017; Jackson et al., 2018; Hindley et al., 2019), and have been detrended using a fourth-order cross-track polynomial filter as in these previous studies. We also (Sect. 4) analyse simulated waves sampled using the measurement geometry of AIRS, for which the wave properties are, by definition, fully known in advance and which, therefore, provide a robust quantitative test.

AIRS is a nadir-sensing instrument and is thus welloptimised geometrically to observe fine horizontal structures. In the horizontal, the scan track has a width of $1780 \mathrm{~km}$, varying in resolution from $\sim 13.5 \mathrm{~km} \times 13.5 \mathrm{~km}$ at nadir to $41 \mathrm{~km} \times 21.4 \mathrm{~km}$ at track edge. The resolution and track width are imposed by the scanning geometry, orbital altitude and hardware design.

In the vertical, the Hoffmann and Alexander (2009) retrieval method uses the 15 and $4.3 \mu \mathrm{m}$ infrared $\mathrm{CO}_{2}$ channels to derive a vertical temperature profile for each measurement footprint. This retrieval is optimised for stratospheric GW studies, with noise and resolution balanced for a vertical resolution between $7-20 \mathrm{~km}$ over the $15-60 \mathrm{~km}$ altitude range. The retrieval switches between a daytime mode, which uses the $15 \mu \mathrm{m}$ channel only, and a night mode which uses both channels; this switch is due to the assumption of local thermodynamic equilibrium required for the underlying scattering calculations being violated during daytime due to solar excitation of $\mathrm{CO}_{2}$ molecules. This switch has an effect on our analysis, manifesting as horizontal stripes of reduced magnitude, seen in the input data at the 33 and $42 \mathrm{~km}$ levels in Figs. 7 and 8, which propagate through to our final output products. Figure 2 of Hindley et al. (2019) illustrates the vertical resolution and retrieval noise as a function of height for a range of regimes.

As such, GWs are often clearly resolved in horizontal cuts through the AIRS measurement volume, but this is usually not the case in the vertical. While an observationally resolved wave may vary over many tens of individual points in the horizontal domain, the entire useful vertical extent of the data is only about 15 data points, many of which overlap in information content. These issues strongly inhibit the use of 3D spectral analysis tools such as the S transform and, as such, make the data set an ideal application for our new approach.

\section{Methods}

The main conceptual tool underpinning this study is the use of phase shifts between spectral features to compute wavelengths. This concept was previously applied to satellite studies of gravity waves by Alexander and Barnet (2007) and Alexander et al. (2008), who used phase shifts between onedimensional $\mathrm{S}$ transforms to compute wavelengths in twodimensional nadir sounder data and one-dimensional limb 

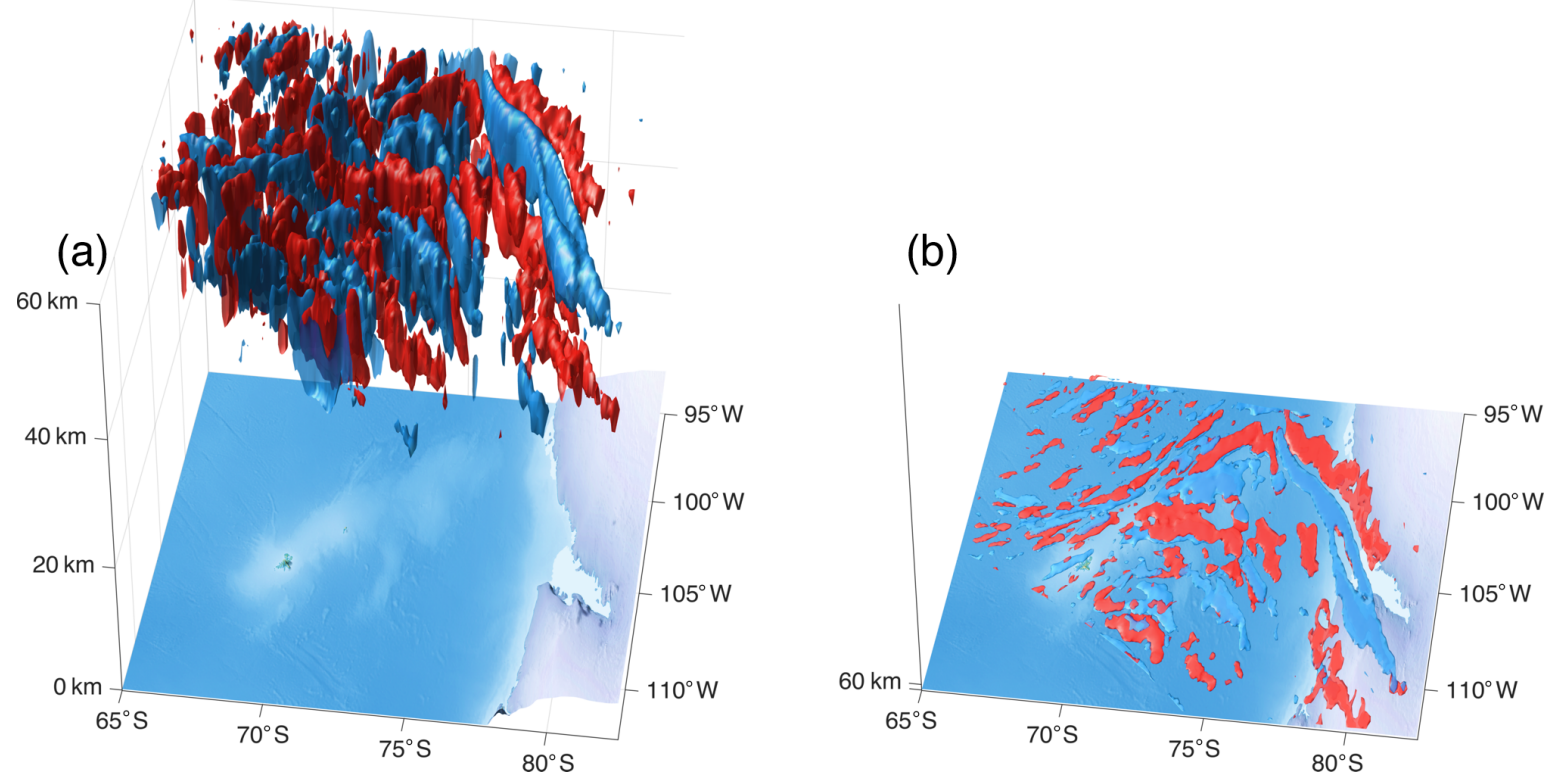

Figure 1. A pair of 3D sketches of temperature perturbations associated with a gravity wave observed on 28 June 2010 over the Southern Ocean, shown (a) with the vertical axis stretched to highlight the wave structure and (b) to true vertical scale. Red/blue isosurfaces enclose the $\pm 3.5 \mathrm{~K} 3 \mathrm{D}$ contours, respectively. Viewing angle and plotted isosurface magnitudes are identical for both panels.

sounder data, respectively. Wright and Gille (2013) expanded upon this work to measure multiple overlapping waves in the same 1D input data signal, and although we do not do so here, the same method could be applied to our new method to determine properties of multiple spectral features at each point in the 3D data volume.

Our new method, which we refer to throughout this study as " $2 \mathrm{D}+1 \mathrm{ST}$ " for brevity, builds instead on the 2D S transform (2D ST) of Hindley et al. (2016) and also uses concepts from the 3D S transform (3D ST) of Wright et al. (2017), as modified by Hindley et al. (2019). The " +1 " here refers to the use of the phase shift for computations made in the vertical dimension. The 2D ST and 3D ST are both multidimensional modifications to the 1D S transform of Stockwell et al. (1996), which allow us, in turn, to extend the phase-shift approach to higher dimensionalities. Although we do not do so here, the same conceptual approach could, for example, be used to identify temporal periods between $3 \mathrm{D}$ volumes of observed or modelled data.

It should also be noted that the ST application of Hindley et al. (2019) used here has a high degree of redundancy, where many more frequencies are analysed at all locations than strictly necessary to compute the transform. However, this is actually advantageous for geophysical data analysis and in particular for the analysis of GW packets, where the amplitude and wavelength of a wave packet may vary in space at scales of less than one wavelength. Despite being computationally slower than lower-redundancy applications of the ST such as the discrete orthonormal S transform (e.g. Wang and Orchard, 2009; Stockwell, 2007), this approach is preferable here as it allows us to reliably measure changes in spectral features at small spatial scales.

\subsection{Data preprocessing}

AIRS data are not uniform in geographic space. In the horizontal domain, this is due to measurement geometry - the measurements are uniform in instrument angular space but distorted in geographic space by the curvature of the Earth - and in the vertical it is due to the relatively lower vertical resolution of the Hoffmann and Alexander (2009) retrieval at the highest and lowest altitudes in the lowermost stratosphere and lower mesosphere.

Since most spectral analyses, including the ST, require regularly gridded data, we must therefore preprocess the AIRS data to make this the case. We do this by first removing altitude levels below $20 \mathrm{~km}$ and above $60 \mathrm{~km}$ - leaving regularly vertically spaced data with a $3 \mathrm{~km}$ level-to-level spacing, since all of the non-regularly spaced levels are outside this range - and then by regridding each level independently onto a regular spatial horizontal grid with the same number of elements (135 along-track by 90 across-track pixels) as the input data but with a uniform inter-point spacing in each direction.

\section{$3.22 \mathrm{D}+1 \mathrm{ST}$ concept}

Figure 2 illustrates the underlying concept of the $2 \mathrm{D}+1 \mathrm{ST}$. Shown on the left of the figure are three separate height levels measured through a large vertical-scale gravity wave, with 
each level separated by a vertical distance of $6 \mathrm{~km}$. Horizontal distance and amplitude units are arbitrary.

For each of these three height levels, we compute the 2D ST $S$ (not shown). This returns a 4D object, where the first two dimensions represent physical distances in the original domain $(x, y)$, and the latter two dimensions represent wavenumber combinations $\left(k_{x}, k_{y}\right)$. For each pair of adjacent levels, we then find the complex cospectrum as follows:

$C_{i, i+1}=S_{i} S_{i+1}^{\dagger}$,

where $S_{i}$ and $S_{i+1}$ are the complex 2D STs of layer $i$ and $i+$ 1 , and the ${ }^{\dagger}$ indicates complex conjugation. Note that, while in this example the levels used to calculate the cospectrum are vertically adjacent, this is not a requirement, and in our analyses below, we use a different vertical step size.

From this cospectrum we then compute a covarying amplitude $A=\sqrt{C_{i, i+1}}$ and a phase difference as follows:

$\Delta \phi=\frac{\operatorname{Im}\left(C_{i, i+1}\right)}{\operatorname{Re}\left(C_{i, i+1}\right)}$,

where Im and Re denote taking the imaginary and real component, respectively. The resulting $A$ and $\Delta \phi$ are both fourdimensional objects, which we collapse to two dimensions by identifying the largest amplitude signal in $\left(k_{x}, k_{y}\right)$ for each $(x, y)$. The resulting two-dimensional $A$ and $\Delta \phi$ thus represent, respectively, the amplitude of the strongest covarying wave-like signal at each pixel in the spatial domain between the two levels and the phase difference between these level pixels.

The right column of Fig. 2 shows the phase differences $\Delta \phi$ between the two level-pairs, with the orange (yellow) solid arrows showing the two height levels contributing to the upper (lower) $\Delta \phi$ map. Phase differences shown are absolute (i.e. unsigned) and shown in units of $\pi$ radians, i.e. a value of 0.5 indicates a phase difference of $\pi / 2$ radians. The choice to make $\Delta \phi$ uniformly positive here is to avoid introducing mathematical signs into our example calculations, and for most practical GW applications, it would instead be sensible to force $\Delta \phi$ to be negative, implying upward wave propagation (Wright et al., 2016, 2017; Ern et al., 2017).

From these phase differences, we can then compute $\lambda_{z}$ between the two levels for each pixel $(x, y)$. A total of two examples of these are shown, indicated by the yellow squares/dashed arrows and by the orange circles/dashed arrows. We consider first the orange example. The pixels highlighted by the orange circle are in a negative node of the wave in the $42 \mathrm{~km}$ layer and in a positive node in the $36 \mathrm{~km}$ layer. For this pair of pixels, the phase difference (orange circle on the right upper layer) is $0.55 \pi$. This implies $\lambda_{z}$ for the wave spanning this pixel pair of $(2 / 0.55 \times 6) 22 \mathrm{~km}$. Following a similar logic for the yellow example, we obtain $\lambda_{z}$ between the lower pair of levels of $18 \mathrm{~km}$. Equivalent estimates of $\lambda_{z}$ can be computed for every pixel on every pair of levels in the data.

\section{$3.32 \mathrm{D}+1 \mathrm{ST}$ calculation procedure}

The above example demonstrates the concept of the $2 \mathrm{D}+1$ ST but is not suitable for general application to data without refinement. In particular, (a) the use of a standard 2D ST will cause the wave amplitude to be significantly underestimated (Wright et al., 2017; Hindley et al., 2016, 2019); (b) when applied to AIRS data retrieved using the method of Hoffmann and Alexander (2009), it is unwise to use adjacent vertical levels in the data for the calculation as they are not sufficiently independent; and (c) if we calculate the 2D ST for all possible horizontal wavenumbers $\left(k_{x}, k_{y}\right)$ at each vertical level, then the total runtime of the procedure can become extremely large.

Therefore, for these reasons and to incorporate the computational improvements made to the 3D ST analysis by Hindley et al. (2019), we use the following procedure to compute our 2D +1 ST outputs for AIRS.

1. We first identify the dominant $1000\left(k_{x}, k_{y}\right)$ combinations in the 3D volume of the data, as described by Hindley et al. (2019). This dramatically reduces the number of independent 2D wavenumber combinations we subsequently need to analyse by removing combinations for which the signal content is very low, which, in turn, very significantly reduces total runtime with no noticeable loss of output quality.

2. Next, we independently compute the 2D ST $S$ of each vertical level in the data for these 1000 dominant wavenumber combinations.

3. Following this, we iterate over height levels, computing and storing the complex cospectrum $C$ between the vertical level above and the vertical level below, i.e. $C_{i-1, i+1}$. This two-vertical-level step increases the independence of the two input levels to the calculation relative to a one-vertical-level step, improving output accuracy. Nominally, this choice prevents us from measuring short $\lambda_{z}$, but as the vertical level spacing in Hoffmann and Alexander (2009) AIRS data is much smaller than the actual vertical resolution of the instrument, no meaningful information is lost.

4. The resulting cospectra are somewhat noisy, due to the high levels of pixel-level noise in the AIRS data relative to typical gravity wave amplitudes. Accordingly, we next average each cospectrum (in 4D) with the cospectra for the levels vertically above and below, using a Gaussian weighting in height which we scale using an analogue to the 3D ST weighting parameter $c$ (Hindley et al., 2016; Wright et al., 2017). In our software implementation, this parameter is scaled such that a value of 1 corresponds to a full width at half maximum (FWHM) of a single $3 \mathrm{~km}$ level, 0.5 indicates 2 levels, etc. This value is fully adjustable and can be used to tune the analysis in the presence of high relative noise. 


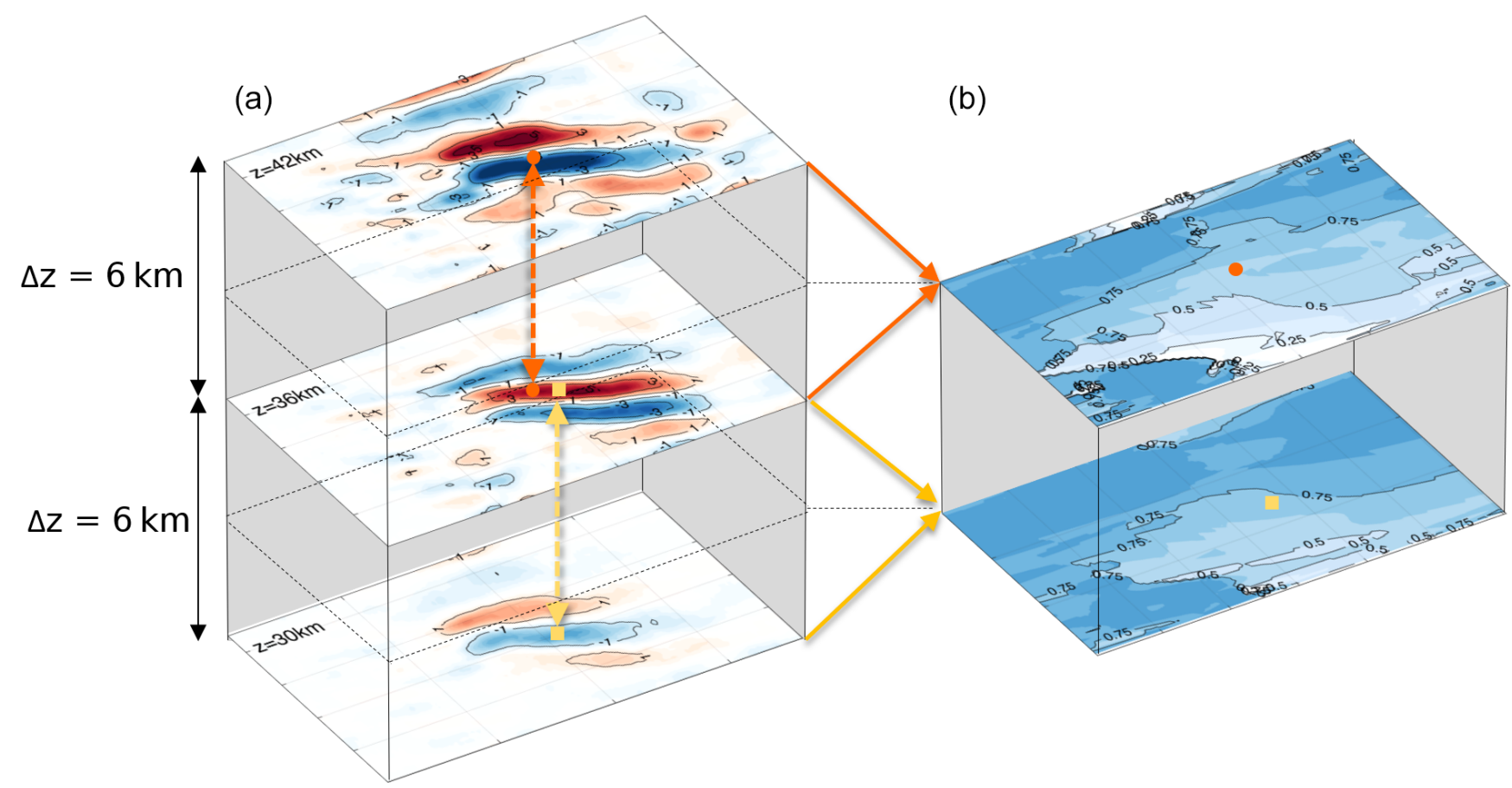

Figure 2. Diagram illustrating the underlying concept of our method. The panel (a) column shows three horizontal cuts through a large vertical-scale gravity wave, with a vertical spacing between levels of $6 \mathrm{~km}$. Values shown at each level are the magnitude of a gravity wave signal, with red indicating a positive magnitude and blue a negative magnitude. All units except for height are arbitrary. The panel (b) column shows cospectral phase differences between the wave structures at each height on the same horizontal domain; these are derived from a $2 \mathrm{D}$ $\mathrm{S}$ transform analysis, with contours showing phase difference in units of $\pi$ radians. Yellow and orange arrows and markers indicate examples of how $\lambda_{z}$ is computed in our analysis and are explained in the main text.

5. Finally, we identify the dominant covarying signal between each pair of vertical layers, $i-1$ and $i+1$, by selecting the wavenumber combinations $\left(k_{x}, k_{y}\right)$ with the largest absolute spectral amplitude $A$ in the cospectrum $C_{i-1, i+1}$, as described in Sect. 3.2. As in Sect. 3.2, we can then compute phase difference $\Delta \phi, \lambda_{z}$ and wave amplitude $A$ for each pixel on each level. We also obtain the horizontal wavenumbers $k_{x}$ and $k_{y}$ from the spectral locations at which the maxima in $A$ were computed. This step incorporates the amplitude-boosting approach of Hindley et al. (2019), which corrects for the amplitude reduction of a standard ST.

In both the simulated and observed analyses below, we use a vertical weighting value of 0.25 , corresponding to a FWHM of $12 \mathrm{~km}$ (i.e. four input data levels). The 2D ST analyses at each level also use a tunable level of $c$ for each horizontal dimension, and in common with previous work, we also set these values to 0.25 .

\section{Simulated data}

We first assess our new technique against a set of simulated waves. This is intended to help us identify any systematic biases or limitations to the technique before moving on to study observed cases.

\subsection{Creating the artificial wave field}

Figure 3 illustrates the method we use to produce our test waves.

We first create an empty volume with the same dimensions as a real AIRS granule by loading a real AIRS granule and setting all temperature values to zero. Before setting the temperatures to zero, the granule is interpolated to a regular spatial grid in the same way as the true AIRS measurements discussed below.

We next create an artificial sinusoid to fill this volume, with a specified temperature amplitude $T^{\prime}$, three-dimensional wavelength $\left(\lambda_{x}, \lambda_{y}, \lambda_{z}\right)$ and rotation $\left(\theta_{x}, \theta_{y}, \theta_{z}\right)$ around a point at the centre of the volume in that dimension (defined as positive in the anti-clockwise direction). We iterate through a wide range of each of these values in our analyses below.

The sinusoid is then weighted with a Gaussian of maximum amplitude 1 and FWHM $1200 \mathrm{~km} \times 950 \mathrm{~km} \times 50 \mathrm{~km}$ centred at the centre of the granule. This is intended to ameliorate any wraparound effects due to the use of fast Fourier transform algorithms in the 2D ST and 3D ST. 


\subsection{Test results}

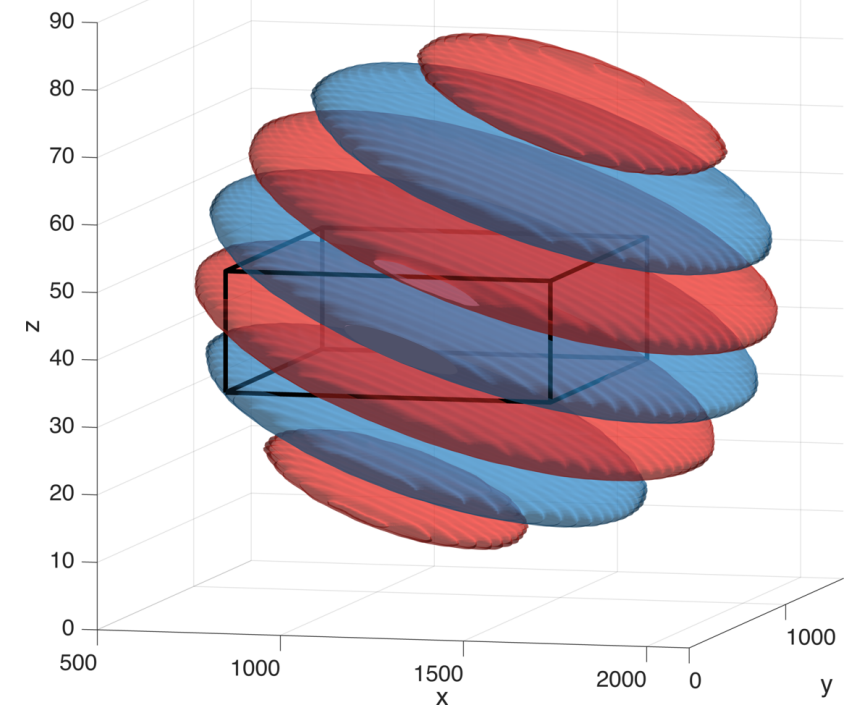

Figure 3. Example figure illustrating the method used for our artificial wave tests. Each wave is a $3 \mathrm{D}$ sinusoid with a specified amplitude, three-dimensional wavelength $\left(\lambda_{x}, \lambda_{y}, \lambda_{z}\right)$ and rotation $\left(\theta_{x}, \theta_{y}, \theta_{z}\right)$ relative to the axes of a 3D Cartesian grid of the same volume as a typical AIRS granule. Red and blue surfaces show 3D contours of a fixed positive (red) and negative (blue) magnitude; values are arbitrary but show the same absolute values for red and blue. Each output parameter is averaged across the region at the centre of the volume indicated by the black box, with dimensions of $800 \mathrm{~km} \times 800 \mathrm{~km} \times 18 \mathrm{~km}$.

We then separately apply the 3D ST and 2D +1 ST to the artificial wave. We do this for both the fully artificial field and also a realistic noise case; this case is produced by adding the detrended $T^{\prime}$ field of a real granule (granule 001 of 8 May 2008) to the fully artificial wave in order to simulate a realistic combined detrending and random noise pattern. This basis granule was empirically selected as one that exhibited no visibly apparent coherent structures that either the $2 \mathrm{D}+1$ ST or 3D ST was likely to identify as a GW. In previous work (Wright, 2010), we have demonstrated that S transform analyses are highly robust to non-systematic noise, and thus, we expect differences between using this granule or another as our basis to be very small.

Finally, to assess the accuracy of the ST outputs, we take the median value of each output field over a $800 \mathrm{~km} \times$ $800 \mathrm{~km} \times 18 \mathrm{~km}$ box at the centre of the wave (shown as a black box in Fig. 3). The median is chosen to better characterise the different effects of the 3D ST and 2D+1 ST on estimated $\lambda_{z}$ in our results (Fig. 41; discussed below), and the subregion is chosen to avoid both wraparound effects and regions where our Gaussian taper has reduced the wave signal to below the noise floor in the realistic-noise cases.
Using the above approach, we estimate wave amplitude $T^{\prime}$, $\lambda_{z}, \lambda_{h}$ and wave propagation angle $\theta$ for a battery of artificial waves. The results of these analyses are shown in Fig. 4 for four methods, namely the 3D ST (orange), the 2D+1 ST (yellow), the 3D ST with realistic noise (blue) and the 2D +1 ST with realistic noise (green).

We have specifically tested the effects on these output variables of variations in input $T^{\prime}, \lambda_{z}$, along-track and acrosstrack wavelengths $\lambda_{x}$ and $\lambda_{y}$, and rotations about the centre of the granule in the $x, y$ and $z$ directions. We omit the rotation in $y$ from Fig. 4 as these results simply reproduce features of rotations in $x$. In all cases, we have varied one of these parameters systematically while holding all others constant at the values shown in the table shown at top right of Fig. 4; these default values were chosen to represent values typical of waves seen in previous AIRS GW studies. Tests with other values of these default parameters (not shown) gave similar relationships between the input and output variables. Values below the spatial resolution of AIRS (specifically, horizontal wavelengths of less than $50 \mathrm{~km}$ and vertical wavelengths below $10 \mathrm{~km}$ ) are omitted.

In general, the results for all four methods are extremely close, and in many cases, particularly for output $\lambda_{h}$ and output propagation angle, they are identical. Rotations in $z$ give the largest effects on all output variables, with the exception of output amplitude (Fig. 4h, n, t); this is because $z$ rotations significantly change the relationship between $\lambda_{z}$ and $\lambda_{h}$ of the artificial wave, with consistent results across all four methods.

In amplitude (Fig. 4a-f), the 2D +1 ST measures very slightly higher amplitudes than the 3D ST in both the nonoise and noise cases, suggesting that the $2 \mathrm{D}+1 \mathrm{ST}$ may be recovering slightly more of the input wave amplitude than the 3D ST. However, this difference is small ( $\sim 5 \%$, at most, and usually less). In all cases, the measured amplitude is significantly lower than that of the input wave (typically by a factor of around 2.5); this is not entirely due to S transform amplitude reduction but is also related to the Gaussian windowing we apply across our range (which will reduce amplitudes at all points away from volume centre) and the choice to generate the wave on the AIRS measurement grid (which will cause us to undersample the theoretical peak magnitudes of the artificial waveform).

The largest differences between tests in the analysis output are seen in output $\lambda_{z}$ when we systematically vary input $\lambda_{z}$ (Fig. 41). In the 3D ST analyses (only the with-noise version is visible as the lines exactly overlap), $\lambda_{z}$ is well recovered at input $\lambda_{z}<30 \mathrm{~km}$, with output $\lambda_{z}$ directly corresponding to this input. Above this value, however, output $\lambda_{z}$ begins to stall at certain wavelengths as input $\lambda_{z}$ increases, with the measured values remaining at fixed output $\lambda_{z}$ values over increasingly long ranges of input $\lambda_{z}$. In contrast, output $\lambda_{z}$ for 

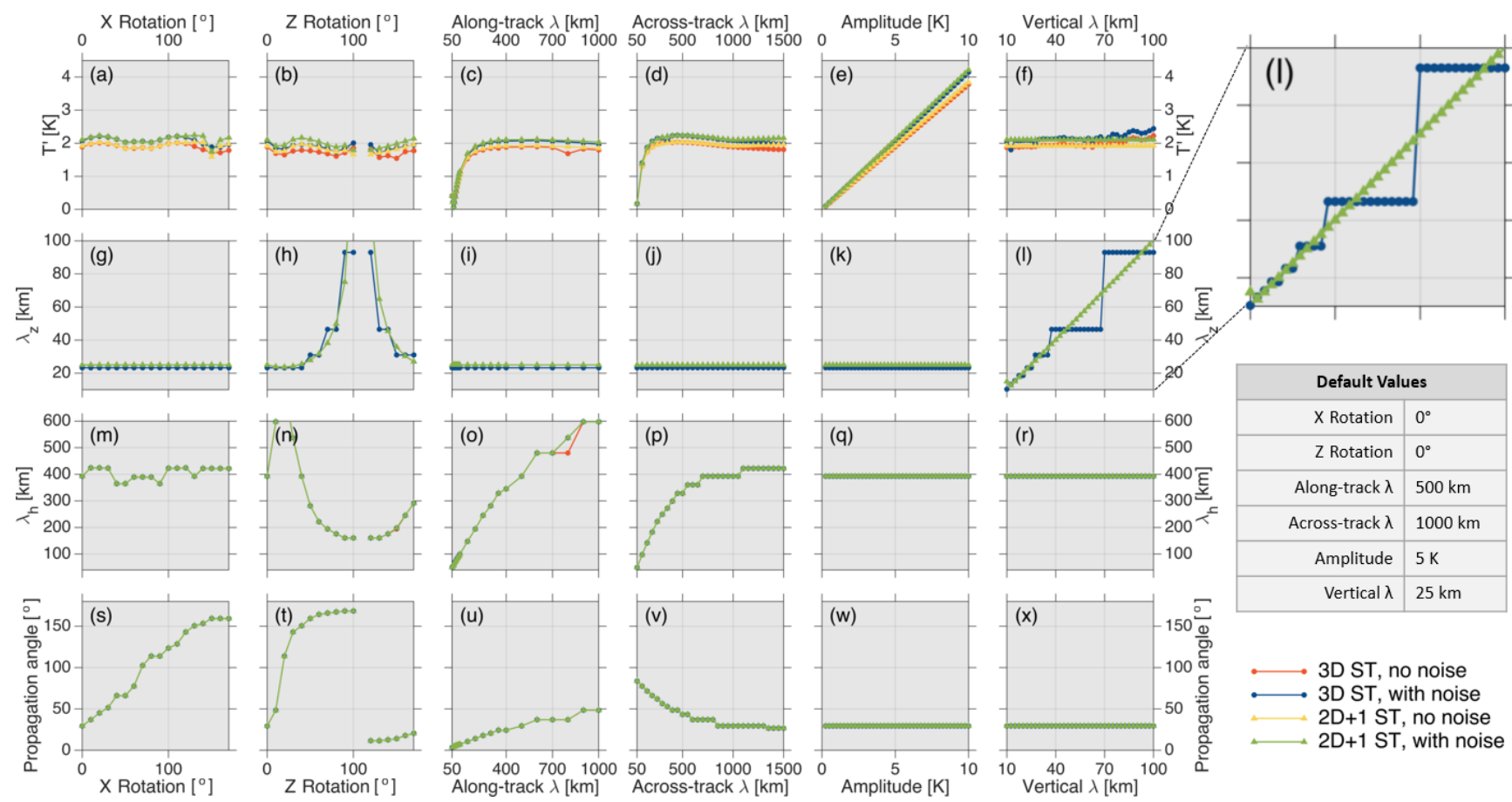

Figure 4. Systematic comparison of the 3D ST and 2D +1 ST for a wide range of input artificial waves. Each panel shows recovered (a-f) wave amplitude, $(\mathbf{g}-\mathbf{l}) \lambda_{z},(\mathbf{m}-\mathbf{r}) \lambda_{h}$ and $(\mathbf{s}-\mathbf{x})$ propagation angle for the four different analyses indicated at bottom right. For each panel, the parameter named at the top and bottom of the column is varied systematically, with all other parameters set to the default values shown in the table at the top right. Note that, in many cases, the results for each method are identical, and for these only the last plotted (2D +1 ST with noise) analysis is visible. Panel (I) is the only test to exhibit significantly different results between the 3D ST and 2D +1 ST and is shown as a magnified inset at the top right.

the $2 \mathrm{D}+1 \mathrm{ST}$ increase smoothly through this range, with no noticeable stepping.

This difference is due to the underlying approach used in the two methods. The 3D ST uses Fourier transform algorithms in the vertical direction, which are inherently limited to resolving integer modes of the data window width. For example, if we were to have 40 points in the vertical, then the 3D ST would only be able to measure wavelengths of $40 / 1$, $40 / 2,40 / 3$, etc. The phase difference approach used in the $2 \mathrm{D}+1 \mathrm{ST}$, by contrast, measures instead the difference in horizontal phase between each selected pair of levels. It is, thus, able to measure a continuous range of $\lambda_{z}$, as we see in Fig. 4l, but with a lower limit (i.e. minimum resolvable wavelength) of twice the level spacing.

As a tradeoff, the $2 \mathrm{D}+1$ ST cannot measure very short vertical waves where the phase change $\Delta \phi$ between the two levels is greater than $\pi$ radians. For AIRS data, such as we are simulating here, this is not a consideration; the vertical resolution of the instrument is coarser than the wavelength at which this problem arises unless inter-level step sizes of greater than 2 are used. However, it must be carefully considered if applying this technique to other data sets or with a different step size. In such cases (not shown), measured vertical wavelengths will be longer than in the underlying data, due to aliasing of phase differences into the $\pm \pi$ range.
For these artificial waves, we, therefore, conclude that the $2 \mathrm{D}+1 \mathrm{ST}$ as implemented here performs equivalently to the 3D ST, with the exception of $\lambda_{z}$ where the 2D+1 ST performs better, provided (not shown) the magnitude of the phase change between the levels we take the cospectrum of is less than $\pi$ radians. This condition is usually true for a two-level step in AIRS data.

\section{Case studies}

We next demonstrate our method on three case study waves, specifically (a) a large Andean mountain wave, (b) a Scandinavian mountain wave and (c) a North American convective wave. These three examples have been selected to represent a range of geophysical challenges, in order to provide a broad test of the 2D $+1 \mathrm{ST}$ approach as compared to the 3D ST.

\subsection{Large Andean mountain wave}

Figures 5a and 6 illustrate our first example, a large orographic wave over South America observed at approximately 06:00 UTC on 6 May 2008. This wave has been used as a baseline test of 3D GW analysis methods in previous studies (Wright et al., 2016, 2017) and, thus, represents a well- 
understood case that is already contextualised within the scientific literature.

The wave is strongly and clearly visible across the whole AIRS altitude range (Fig. 6a,h), with vertically slanted phase fronts aligned to indicate a source on the west coast of South America. It has a very large amplitude, with magnitudes peaking at $> \pm 15 \mathrm{~K}$ (note that the colour scale saturates at $\pm 10 \mathrm{~K}$ ) and is angled across the AIRS scan track but with a significant along-track component. It is, thus, highly suited to study using AIRS data.

For all fields other than the input temperature perturbations, we have set voxels (i.e. 3D pixels) with amplitudes $<$ $2.7 \mathrm{~K}$ after smoothing with a $5 \times 5$ voxel horizontal-domainonly boxcar to zero, in order to remove noise-dominated regions and focus on resolved wave structure. The boxcar size and amplitude cutoff have been selected empirically but can also be considered on physical grounds. The smoothing ensures extremely localised variations do not produce distracting gaps in plotted regions, while the $2.7 \mathrm{~K}$ value is approximately twice the AIRS retrieval noise floor at the central height in our data range, and thus, this approach will retain packet-edge locations where the smoothing would average these values to below the real noise floor. Most large-scale studies of GWs in AIRS data (e.g. Ern et al., 2017; Hindley et al., 2020; Perrett et al., 2021) impose similar minimumamplitude criteria before including a given voxel in their analyses.

In 3D ST output (Fig. 6), the wave is clearly visible, with a well-defined region of high wave amplitude resolved over Chile, Argentina and the surrounding seas. We also see associated patches of well-defined $\lambda_{h}$. The same wave was studied using an earlier version of our 3D ST implementation by Wright et al. (2017), and only small differences are visible between their old and our revised 3D ST outputs, primarily the higher measured amplitudes.

The $2 \mathrm{D}+1 \mathrm{ST} \lambda_{h}$ is very similar to that obtained from the 3D ST, with the exception of a region of long $\lambda_{h}$ at low altitude in the northern part of the along-track cut (Fig. 6m). This region both exhibits very low wave amplitude relative to the rest of the wave shown and is only sporadically filled from voxel to voxel in both analyses; thus, both analyses are likely largely fitting to background noise rather than true wave signals in this region despite our output filtering.

Larger differences in output are seen for amplitude and $\lambda_{z}$. Amplitude is significantly more spatially localised for the 2D + 1 ST than for the 3D ST, likely arising from the calculation of amplitude independently for each level pair, and this is discussed further in the general case below.

The $\lambda_{z}$ in Fig. $6 \mathrm{~d}, \mathrm{~g}, \mathrm{k}$ and $\mathrm{n}$ exhibits the largest difference, consistent with our intentions behind developing the 2D + 1 ST approach. The 3D ST estimates are limited to Fourier modes of the vertical window, which in practice allow only two unique values across the whole domain in both the horizontal and vertical slices, specifically 20 and $13 \mathrm{~km}$. The measured $\lambda_{z}$ for every voxel is one of these two val- ues. This effect was also visible in Wright et al. (2017). The 2D+1 ST output, on the other hand, exhibits a much broader spectrum of $\lambda_{z}$, with values distributed across a continuum. Smooth variations in $\lambda_{z}$ can be clearly seen in both the mapped (Fig. 6g) and sliced (Fig. 6n) fields, as would be expected in the real atmosphere.

As examples of features in the horizontal plane which can be identified in the 2D+1 ST output but not the 3D ST output, we clearly resolve a region of longer $\lambda_{z}$ over the Golfo San Jorge (San Jorge Gulf; $67^{\circ} \mathrm{W}, 45^{\circ} \mathrm{S}$ ) and a region of shorter $\lambda_{z}$ over Tierra del Fuego $\left(69^{\circ} \mathrm{W}, 55^{\circ} \mathrm{S}\right)$. In the vertical plane, we see a smooth increase in $\lambda_{z}$ across the height range, suggesting lengthening of $\lambda_{z}$ with height. This effect can be discerned from the 3D ST output as a discontinuity between a low $\lambda_{z}$ region below $30 \mathrm{~km}$ altitude and a longer $\lambda_{z}$ region above this, but the rate of growth can be clearly quantified in the $2 \mathrm{D}+1 \mathrm{ST}$ version. We can also determine that growth occurs gradually across the entire range rather than discontinuously across a narrower height range around $30 \mathrm{~km}$, which was not possible with 3D ST output alone. Even in central altitude regions with $\sim 39 \mathrm{~km}$ altitude, where AIRS data have their best resolution and lowest noise, the $2 \mathrm{D}+1$ shows more realistic variations in $\lambda_{z}$ with horizontal distance.

For this case study, therefore, we conclude that $\lambda_{h}$ data quality is largely unchanged, and that $\lambda_{z}$ data quality is significantly improved. Amplitude differs between the two versions, but our comparison does not provide sufficient information to identify if this change is an improvement or a reduction in output quality.

\subsection{Scandinavian mountain wave}

Our second example, Figs. 5b and 7, is an orographic wave over Scandinavia observed shortly after 12:00 UTC on 13 January 2007. This wave is also clearly visible in AIRS data but has a much smaller amplitude than our first example and also covers a smaller geographic area, thus providing a more exacting test.

As with the Andean case above, the wave is made up of clearly defined parallel phase fronts, sloping upwards at an angle to the AIRS scan track. Phase front magnitudes in the raw AIRS data are larger at low altitude, with a slight reduction in amplitude above $40 \mathrm{~km}$ altitude. Amplitude measurements from both the 3D ST and 2D+1 ST are consistent with this, with maxima at around $35 \mathrm{~km}$ altitude. These maxima are tightly spatially localised around the southern half of Norway and western parts of central Sweden.

Both analyses show minor flaws in their output amplitude field, with the 3D ST producing a patch of extremely large amplitudes at the top of the plotted altitude range and the $2 \mathrm{D}+1$ ST exhibiting two horizontal stripes, at $\sim 33$ and $\sim$ $42 \mathrm{~km}$ altitude. In the latter case, these stripes are caused by similar horizontal stripes visible in the input data at these altitudes (Fig. 7h), which the 3D ST is able to compensate 
(a)

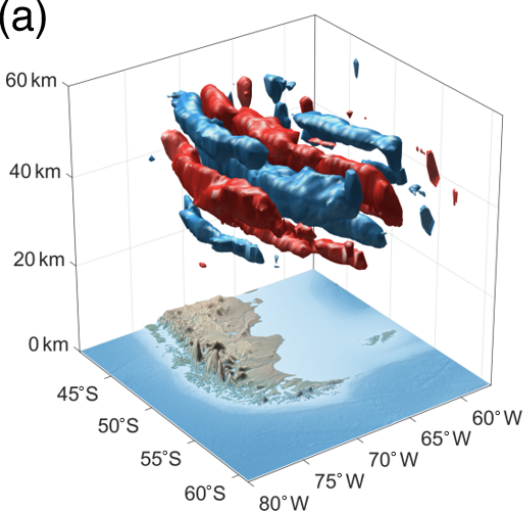

(b)

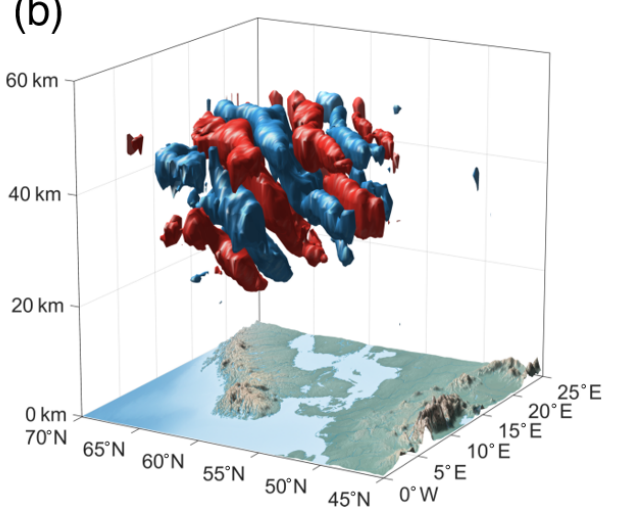

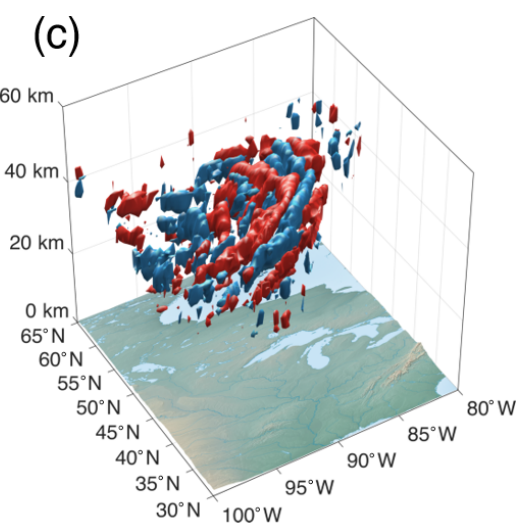

Figure 5. A trio of 3D sketches of the three waves used as case studies; amplitudes have been omitted as these are shown in cross section below (Figs. 6-8). Viewing angles have been selected to best highlight the 3D phase structure of each case.

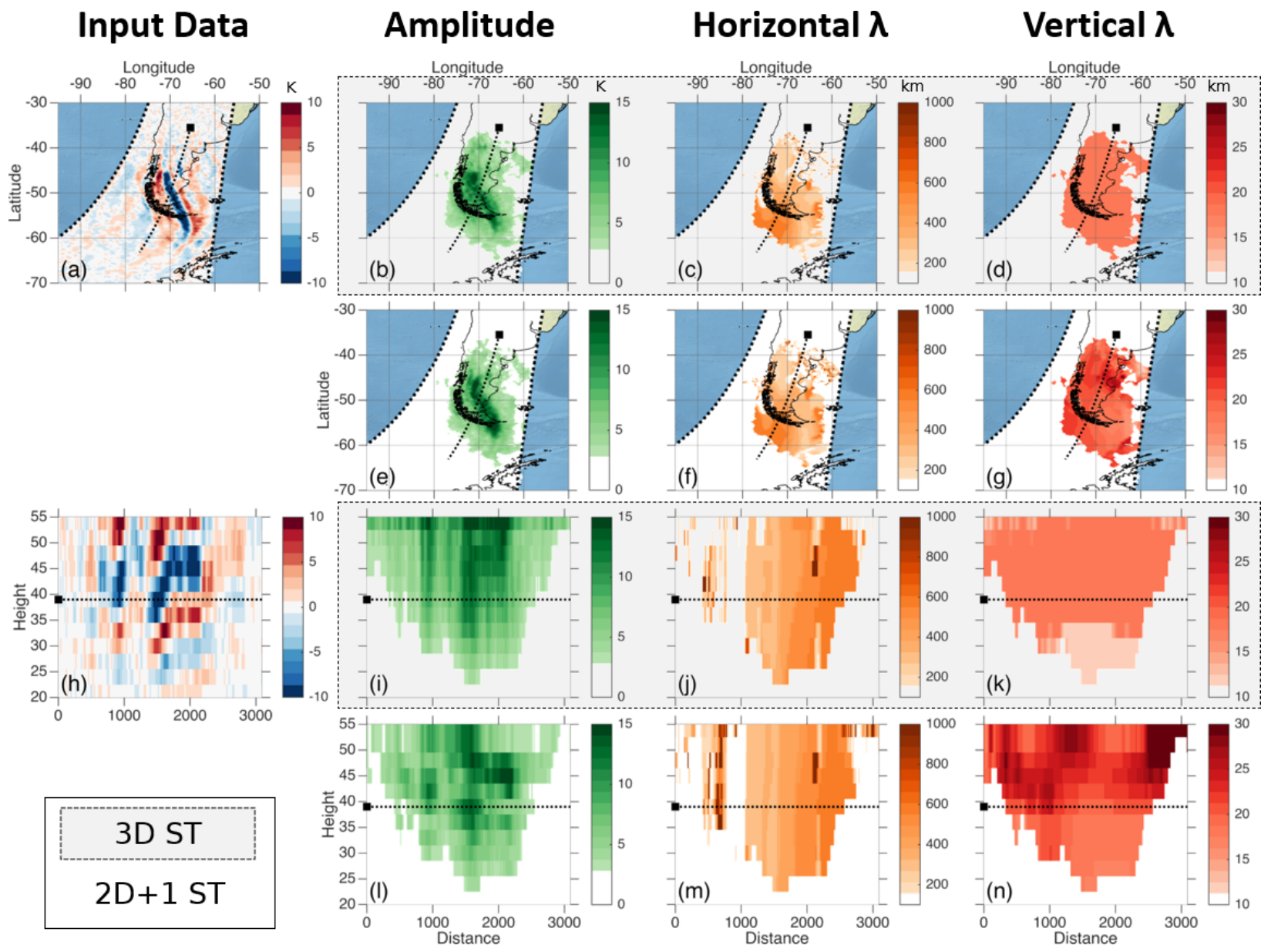

Figure 6. Comparison of 3D ST and 2D +1 ST estimates of GW parameters for a large orographic gravity wave observed in May 2008 over South America. Panels $(\mathbf{a}, \mathbf{h})$ show the original temperature perturbation data as a map at $39 \mathrm{~km}$ altitude and a vertical cut along the instrument track, respectively; dotted lines show the location of the other set of panels, with a black square indicating zero distance on the lower panel. (b-d, i-k) 3D ST estimates, $(\mathbf{e}-\mathbf{g}, \mathbf{l}-\mathbf{n}) 2 \mathrm{D}+1$ ST estimates of $(\mathbf{b}, \mathbf{e}, \mathbf{i}, \mathbf{l})$ wave amplitude, $(\mathbf{c}, \mathbf{f}, \mathbf{j}, \mathbf{m}) \lambda_{h}$ and $(\mathbf{d}, \mathbf{g}, \mathbf{k}, \mathbf{n}) \lambda_{z}$. Units for each panel are shown above the colour bar at the top of each row. Data have been boxcar smoothed by 5 voxels in each direction in the horizontal plane before plotting to reduce visible noise. Regions with smoothed output amplitudes below $2.7 \mathrm{~K}$ have been removed from all panels, except $(\mathbf{a}, \mathbf{h})$, to focus on wave features only. 


\section{$\begin{array}{llll}\text { Input Data } & \text { Amplitude } & \text { Horizontal } \boldsymbol{\lambda} & \text { Vertical } \boldsymbol{\lambda}\end{array}$}

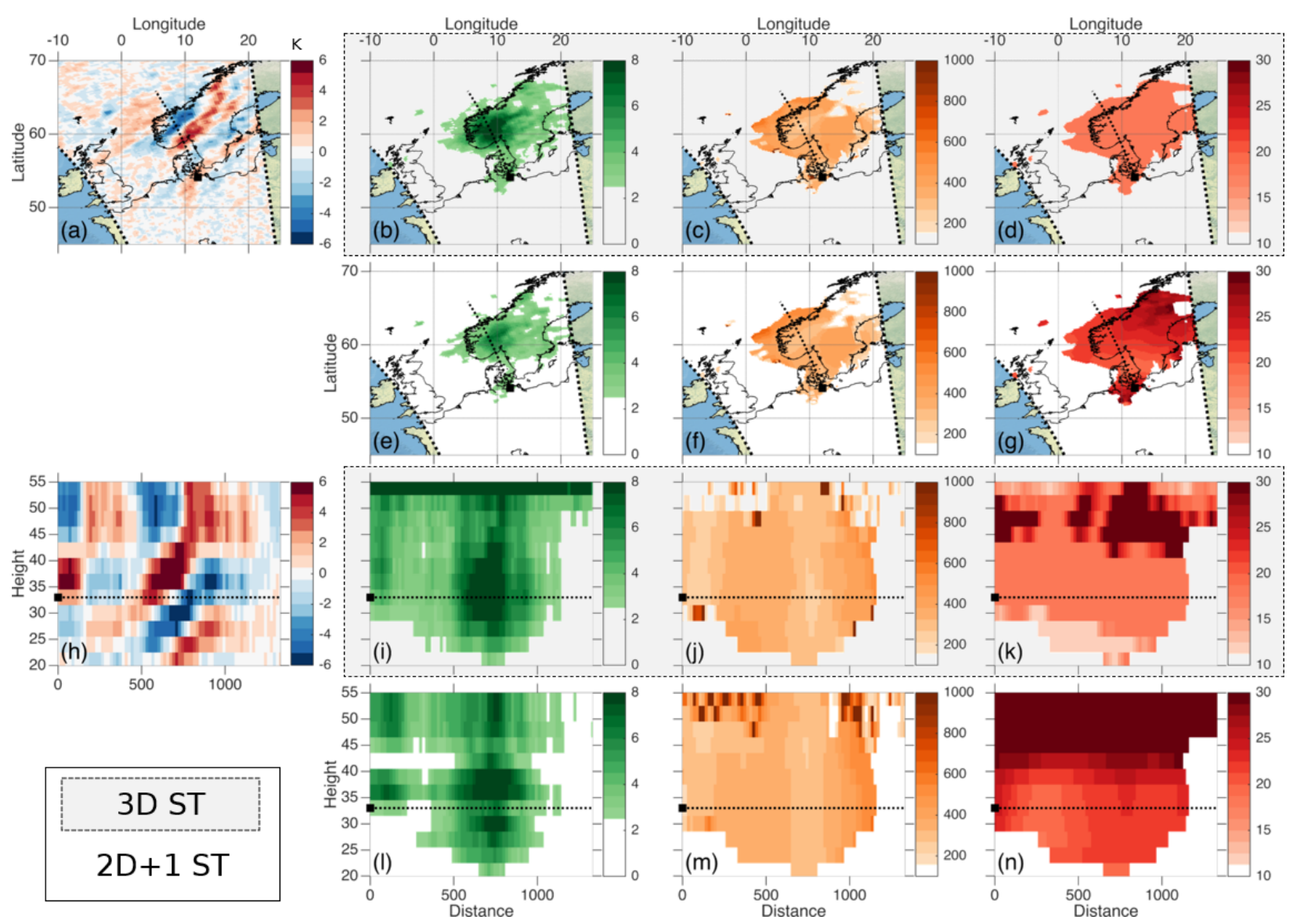

Figure 7. As in Fig. 6 but for an orographic wave observed over Scandinavia in January 2007 and with horizontal cuts taken at $33 \mathrm{~km}$ altitude. Note that in panel (n) $\lambda_{z}$ at high altitudes very significantly saturates the colour scale; this is discussed further in the text.

for by implicitly using information from surrounding height levels, but which the $2 \mathrm{D}+1 \mathrm{ST}$ is unable to compensate for as individual level pairs are used. We, therefore, argue that the 2D+1 ST output is more faithful to the input data, while the 3D ST output is likely a more accurate description of the true atmospheric feature in this individual case. The ultimate origin of these stripes is due to the use of the daytime rather than nighttime retrieval, as discussed in Sect. 2.

Both analyses see the same along-track pattern of $\lambda_{h}$, with longer $\lambda_{h}$ between $200-600 \mathrm{~km}$ along-track from our origin (black square), then shorter $\lambda_{h}$ for around $200 \mathrm{~km}$, before increasing again until the edge of the wave packet is reached. As with the previous example, the 2D $+1 \mathrm{ST}$ has a tendency to produce longer estimates of $\lambda_{h}$ in regions of low amplitude, i.e. without significant wave activity - in this case, this is most visible at the top left of Fig. 7m, where the input temperature variations have become near-vertical and amplitudes are at their lowest above-cutoff values.

The 3D ST $\lambda_{z}$ for this example exhibits three unique values increasing with height, except for a small layer at the top of the data. The $2 \mathrm{D}+1 \mathrm{ST}$ output again exhibits a continuum of values, increasing steadily across the height range. At heights below $40 \mathrm{~km}, 2 \mathrm{D}+1 \mathrm{ST}$ output $\lambda_{z}$ across the whole height range is slightly higher than the $3 \mathrm{D} \mathrm{ST}$, but this difference is consistent with values obtained from a visual examination of Fig. $7 \mathrm{~h}$ and, thus, reflects a better estimate of the true wave properties.

Estimated values of $\lambda_{z}$ increase rapidly at the top of the altitude range, significantly saturating the colour scale to reach maximal values above $50 \mathrm{~km}$ altitude of $\sim 120 \mathrm{~km}$ at alongtrack distances between 500-1000 and $>150 \mathrm{~km}$ elsewhere. These values are extremely large but consistent with the input data (Fig. 7h) where the phase fronts are near-vertical at these altitudes. This may be a real effect, or it may be an artefact of the significantly reduced instrument vertical resolution at these altitudes (see, e.g., Fig. 2 of Hindley et al., 2019). However, the same effect is not seen in our other case studies and, furthermore, is seen here as a continuous extension of the phase fronts present in the lower part of the stratosphere (note that our data are detrended independently at each height level), suggesting at least some contribution from the real atmosphere. 
Input Data

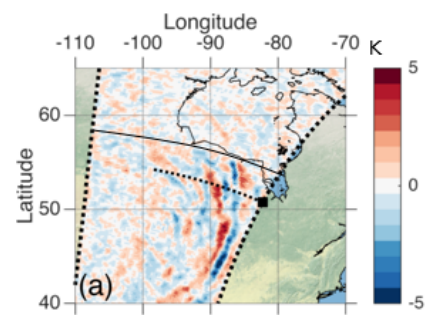

Amplitude
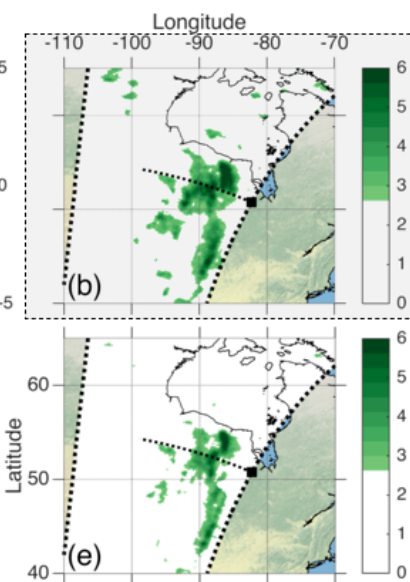

(e)

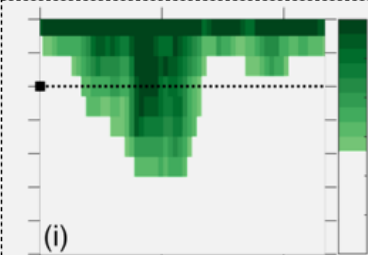

(h)
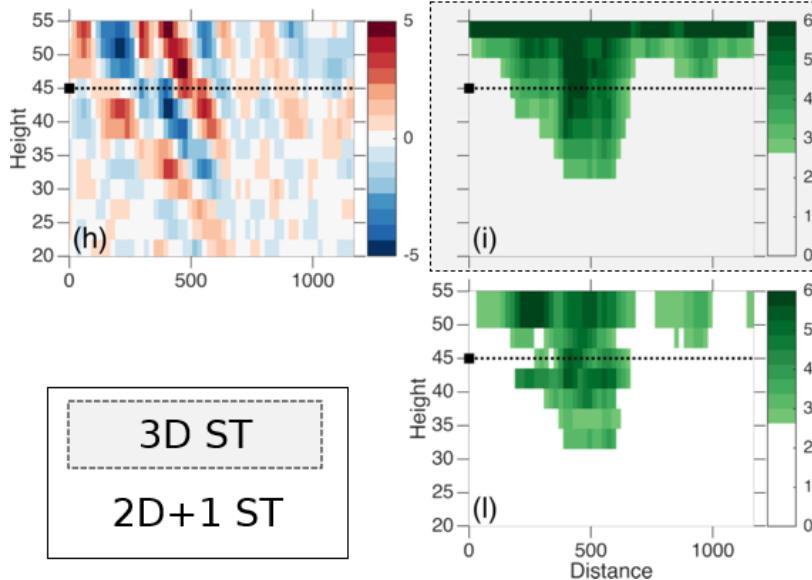

Horizontal $\lambda$
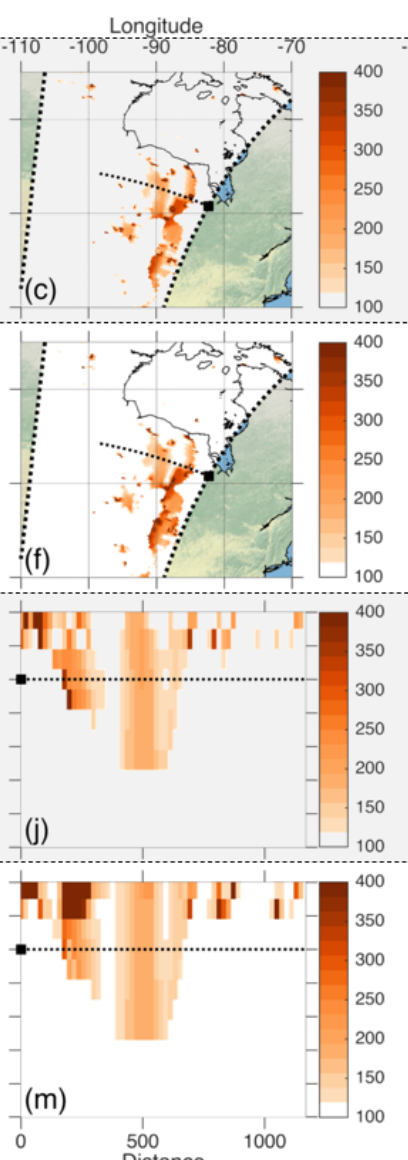

Vertical $\lambda$
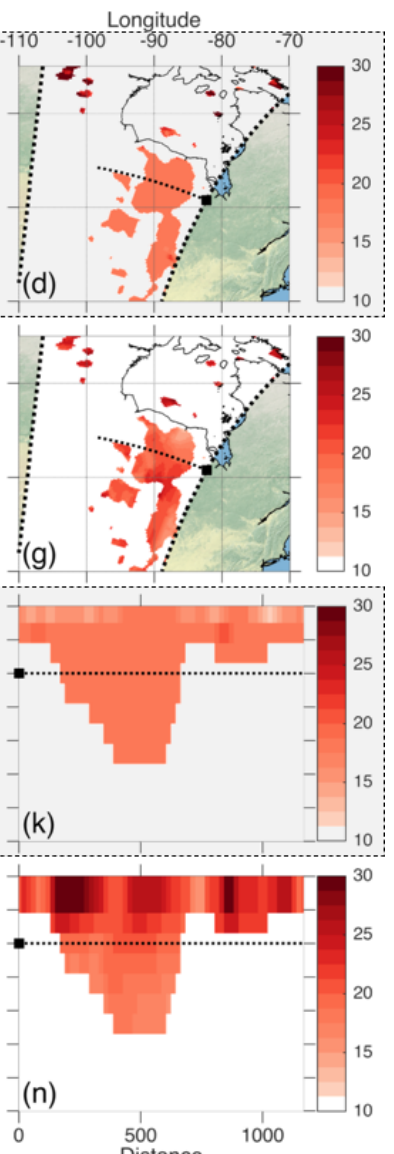

Figure 8. As in Fig. 6 but for a convective wave observed over North America in July 2008 and with horizontal cuts taken at $45 \mathrm{~km}$ altitude. A discontinuity is visible in some panels between a region on the equatorward side of the maps and a region on the poleward side; this is due to a switch between the nighttime and daytime retrievals, which have very different noise characteristics.

Finally, as with the previous example, with the $2 \mathrm{D}+1 \mathrm{ST}$ we can measure structural variations in the $\lambda_{z}$ field which were invisible to the 3D ST. In this case, a previously invisible feature is a reduction in $\lambda_{z}$ between the main Scandinavian ridgeline $\left(\sim 17^{\circ} \mathrm{E}, 67^{\circ} \mathrm{N}\right)$ and the lower-lying regions of Sweden heading towards Våstergötland and the Øresund $\left(\sim 10^{\circ} \mathrm{E}, 56^{\circ} \mathrm{N}\right)$, which, while nominally visible as a step change in the 3D ST output, follows a clear gradient in the 2D+1 ST output. Indeed, a north-south gradient in $\lambda_{z}$ is clearly seen across the whole of southern Sweden in the $2 \mathrm{D}+1 \mathrm{ST}$ output, which is reduced to a single switch of mode in the 3D ST.

\subsection{North American convective wave}

Our final example, Figs. $5 \mathrm{c}$ and 8 , is a convection-generated wave observed between 08:00 and 09:00 UTC over North America on 8 July 2008. This wave is a much more challenging test of our method than the previous two case studies for the following four reasons:
1. The wave has a maximum amplitude of $\sim 5 \mathrm{~K}$, which is small compared to the previous examples and, thus, closer to the instrument noise floor.

2. The wave is curved about an arc rather than being well aligned with the instrument frame of reference and, hence, changes rapidly in along-track and across-track wavelengths in the instrument frame of reference.

3. The wave is close to the edge of the AIRS swath in a region where across-track horizontal resolution is around a factor of 2 below that at track centre.

4. The wave is close to the day-night terminator, where the Hoffmann and Alexander (2009) retrieval switches mode, and thus, measurements of this wave are potentially complicated by a discontinuity in noise level and spatial resolution only a few $100 \mathrm{~km}$ away from the wave centre. This manifests itself in Fig. 8a as a reduction in the input temperature range poleward of a 
cross-track line approximately along the southern edge of Hudson Bay (indicated by a black solid line).

The wave is visible in Fig. 8a as a series of arcs on the eastern side of the AIRS swath, with radii centred at a point somewhere between Calgary and Medicine Hat, both in Alberta - specifically, the curvature of the arcs suggests the origin to be around $112^{\circ} \mathrm{W}, 51^{\circ} \mathrm{N}$, which is slightly off the left side of the map. Convective cloud data from the AIRS $8.1 \mu \mathrm{m}$ channel (Fig. S1 in the Supplement) show evidence of convective activity in this region throughout this day and the day before, and analysis of the relative 2D horizontal wavelengths in the along-track and cross-track directions (not shown) further confirm that the wave is likely to be radiating from around this location. Our maps are shown at the $45 \mathrm{~km}$ altitude level, but at lower stratospheric altitudes, the wave is challenging to distinguish from background without prior knowledge of its location and is at the very noise limit of AIRS data.

Consistent with this difficulty, amplitudes in both analyses are largest at altitudes above $35-40 \mathrm{~km}$. Above-background amplitudes are also tightly spatially localised around the narrow observed wavefronts. There is perhaps some visual evidence in Fig. 8a that the wave arc may continue at much lower amplitudes poleward of the terminator, but this is not visible at above-cutoff levels in the output amplitude data; visual analysis of the full below-noise amplitude data fields (not shown) also do not show evidence that these features are detected as waves by either analysis method.

$\lambda_{h}$ is near-identical for the two analyses, with the maps showing a region of $\lambda_{h} \sim 200-350 \mathrm{~km}$ along the wave front and the slices through the wave showing a tight region of $\lambda_{h} \sim 200 \mathrm{~km}$ at all heights around the magnitude peak. This band extends throughout the entire height range from 20 to $60 \mathrm{~km}$; since each level pair in the $2 \mathrm{D}+1 \mathrm{ST}$ analysis is treated independently, this demonstrates that, while amplitude and discernibility from background of the wave are poor at low altitudes, ST-based analyses are still capable of measuring $\lambda_{h}$ even against a high relative noise floor.

Finally, and consistently with all previous tests, we see $\lambda_{z}$ steadily increasing across the height range, with a unique measured value $\lambda_{z}$ for the 3D ST over almost the whole data extent and a continuum for the $2 \mathrm{D}+1 \mathrm{ST}$.

\section{Summary, discussion and conclusions}

In this study, we have described and implemented a new spectral analysis method (the 2D+1 ST) for the characterisation of wave-like signals in three-dimensional data. This method is particularly well suited to data which are fine in two dimensions but coarse in the third, which is very often the case for satellite measurements of the Earth's atmosphere, and to data where the feature size to be extracted is large relative to the total domain length. This method uses a two-dimensional Stockwell transform (Stockwell et al., 1996; Hindley et al., 2016) in the well-resolved twodimensional plane, with wavelengths in the coarse third dimension computed from phase differences between $\mathrm{S}$ transformed two-dimensional levels.

We have tested this new method on artificial waves, both with and without noise, and on observed case study waves in real data from NASA's AIRS instrument. In all cases, the $2 \mathrm{D}+1 \mathrm{ST}$ is almost as identically capable as the $3 \mathrm{D}$ ST for determining fine-dimension (usually horizontal) wave structures and much better suited to characterising coarsedimension (usually vertical) structures. This improvement is primarily because the phase difference approach used is not restricted to Fourier modes of the domain size.

The $2 \mathrm{D}+1 \mathrm{ST}$ wave amplitudes are equivalent in magnitude to those obtained from the 3D ST and more responsive to local variations in input wave amplitude. This is, in principle, superior to the 3D ST, as the inputs are better reflected in the outputs. However, in real cases it may represent a slight degradation in the direct usefulness of results, as the broader contextual information used in the 3D ST can help to ameliorate localised data quality deficiencies in the input field. This is, however, a small effect.

Although we do not discuss it in the body of the article, the $2 \mathrm{D}+1 \mathrm{ST}$ is also computationally slower than the 3D ST due to both the need to (1) analyse each level with an individual 2D ST and (2) to take phase differences between levels, with the latter having a larger overall impact on runtime. This may affect the choice of which algorithm to use when considering large volumes of data. For the three case studies considered, the 2D+1 ST typically takes twice the runtime of the equivalent 3D ST on the two-core system used for this study; as the $S$ transform parallelises well, this relative increase may be smaller on nodes with more available cores. The runtime difference could be further ameliorated by reducing the number of individual frequency combinations considered - at a small cost to output accuracy.

We conclude, therefore, that the $2 \mathrm{D}+1 \mathrm{ST}$, in general, outperforms the 3D ST for measuring waves in data with at least one coarse dimension, a category which includes almost all current-generation Earth observation satellite data. In particular, the $2 \mathrm{D}+1 \mathrm{ST}$ has two major advantages over the 3D ST for application to satellite observations such as AIRS and similar nadir sounders, viz:

1. It allows us to identify spatial variations in measured $\lambda_{z}$ that may have physical significance for the geophysics of the wave environment. In our case studies, we have been able to identify regional changes in $\lambda_{z}$ that were not visible or only weakly visible to the 3D ST, e.g. finding longer wavelengths over the Golfo San Jorge and shorter wavelengths over Tierra del Fuego (Fig. 6g) and a general north-south reduction in wavelength over the west of Sweden (Fig. 7g), in addition to a generally much better characterisation of $\lambda_{z}$ growth with height. 
2. It is much more suited to the measurement of extremely long vertical wavelengths, including those that are longer than the visible length of the wave in the measured data. This capability is demonstrated both for test waves (Fig. 41) and practically at high altitudes in our Scandinavian case study. This is of significant benefit for measuring portions of the gravity wave spectrum that are extremely hard to observe, including, for example, hypothesised extremely long $\lambda_{z}$ waves which may act to transport large quantities of surface momentum directly to the upper atmosphere (Liu et al., 2013; Ern et al., 2018).

In future work, we intend to apply this new technique to other satellite and ground-based data sets, with the dual aims of better characterising internal variability in the stratospheric and mesospheric gravity wave spectrum and of identifying and quantifying the effects of long $\lambda_{z}$ waves on upperatmospheric dynamics.

Code and data availability. All code produced for and data used in this study, with the exception of the topography and surface imagery data sets used to contextualise Figs. 1 and 58 , have been archived with Zenodo and can be accessed via https://doi.org/10.5281/zenodo.4569067 (Wright, 2021).

Supplement. The supplement related to this article is available online at: https://doi.org/10.5194/amt-14-5873-2021-supplement.

Author contributions. MJA provided the original suggestion for the work. CJW produced the figures, wrote the initial draft and developed an initial algorithmic approach in collaboration with MJA and LAH. NPH heavily revised the algorithmic approach to be more computationally efficient and significantly more accurate. LH produced and supplied the AIRS data used in our examples. All authors contributed to methodological development and to the final paper.

Competing interests. The authors declare that they have no conflict of interest.

Disclaimer. Publisher's note: Copernicus Publications remains neutral with regard to jurisdictional claims in published maps and institutional affiliations.

Acknowledgements. Corwin J. Wright was supported by a Royal Society University Research Fellowship (grant no. UF160545) and NERC (grant nos. NE/R001391/1 and NE/S00985X/1). Neil P. Hindley was also supported by the latter two grants. M. Joan Alexander and Laura A. Holt were supported, in part, by the Wave-induced Atmospheric Variability Enterprise (WAVE), a NASA Heliophysics DRIVE Science
Center (grant no. 80NSSC20K0628) and by NASA (grant no. 80NSSC18K0069). Laura A. Holt was further supported by NASA (grant no. 80NSSC18K0768). We also acknowledge the vital underpinning discussions at and within the context of the "New Quantitative Constraints on Orographic Gravity Wave Stress and Drag", an international team project supported by the International Space Science Institute, and during an NorthWest Research Associates (NWRA)-funded visit to Boulder, Colorado, by Corwin J. Wright to collaborate on developing an initial approach with M. Joan Alexander and Laura A. Holt.

Financial support. This research has been supported by the Natural Environment Research Council (grant nos. NE/R001391/1 and NE/S00985X/1), the Royal Society (grant no. UF160545), the NASA Heliophysics DRIVE Science Center (grant no. 80NSSC20K0628), and NASA (grant nos. 80NSSC18K0069 and 80NSSC18K0768).

Review statement. This paper was edited by Jorge Luis Chau and reviewed by two anonymous referees.

\section{References}

Alexander, M. J. and Barnet, C.: Using Satellite Observations to Constrain Parameterizations of Gravity Wave Effects for Global Models, J. Atmos. Sci., 64, 1652-1665, https://doi.org/10.1175/jas3897.1, 2007.

Alexander, M. J., Gille, J., Cavanaugh, C., Coffey, M., Craig, C., Eden, T., Francis, G., Halvorson, C., Hannigan, J., Khosravi, R., Kinnison, D., Lee, H., Massie, S., Nardi, B., Barnett, J., Hepplewhite, C., Lambert, A., and Dean, V.: Global estimates of gravity wave momentum flux from High Resolution Dynamics Limb Sounder observations, J. Geophys. Res., 113, D15S18, https://doi.org/10.1029/2007jd008807, 2008.

Aumann, H., Chahine, M., Gautier, C., Goldberg, M., Kalnay, E., McMillin, L., Revercomb, H., Rosenkranz, P., Smith, W., Staelin, D., Strow, L., and Susskind, J.: AIRS/AMSU/HSB on the aqua mission: design, science objectives, data products, and processing systems, IEEE T. Geosci. Remote, 41, 253-264, https://doi.org/10.1109/tgrs.2002.808356, 2003.

Blumstein, D., Chalon, G., Carlier, T., Buil, C., Hebert, P., Maciaszek, T., Ponce, G., Phulpin, T., Tournier, B., Simeoni, D., Astruc, P., Clauss, A., Kayal, G., and Jegou, R.: IASI instrument: technical overview and measured performances, in: Infrared Spaceborne Remote Sensing XII, edited by: Strojnik, M., Society of Photo-Optical Instrumentation Engineers, Denver, Colorado, USA, SPIE, https://doi.org/10.1117/12.560907, 2004.

Chahine, M. T., Pagano, T. S., Aumann, H. H., Atlas, R., Barnet, C., Blaisdell, J., Chen, L., Divakarla, M., Fetzer, E. J., Goldberg, M., Gautier, C., Granger, S., Hannon, S., Irion, F. W., Kakar, R., Kalnay, E., Lambrigtsen, B. H., Lee, S.-Y., Marshall, J. L., Mcmillan, W. W., Mcmillin, L., Olsen, E. T., Revercomb, H., Rosenkranz, P., Smith, W. L., Staelin, D., Strow, L. L., Susskind, J., Tobin, D., Wolf, W., and Zhou, L.: AIRS, B. Am. Meteorol. Soc., 87, 911-926, https://doi.org/10.1175/bams-87-7911, 2006. 
Ern, M., Hoffmann, L., and Preusse, P.: Directional gravity wave momentum fluxes in the stratosphere derived from highresolution AIRS temperature data, Geophys. Res. Lett., 44, 475485, https://doi.org/10.1002/2016g1072007, 2017.

Ern, M., Trinh, Q. T., Preusse, P., Gille, J. C., Mlynczak, M. G., Russell III, J. M., and Riese, M.: GRACILE: a comprehensive climatology of atmospheric gravity wave parameters based on satellite limb soundings, Earth Syst. Sci. Data, 10, 857-892, https://doi.org/10.5194/essd-10-857-2018, 2018.

Hilton, F., Armante, R., August, T., Barnet, C., Bouchard, A., Camy-Peyret, C., Capelle, V., Clarisse, L., Clerbaux, C., Coheur, P.-F., Collard, A., Crevoisier, C., Dufour, G., Edwards, D., Faijan, F., Fourrié, N., Gambacorta, A., Goldberg, M., Guidard, V., Hurtmans, D., Illingworth, S., Jacquinet-Husson, N., Kerzenmacher, T., Klaes, D., Lavanant, L., Masiello, G., Matricardi, M., McNally, A., Newman, S., Pavelin, E., Payan, S., Péquignot, E., Peyridieu, S., Phulpin, T., Remedios, J., Schlüssel, P., Serio, C., Strow, L., Stubenrauch, C., Taylor, J., Tobin, D., Wolf, W., and Zhou, D.: Hyperspectral Earth Observation from IASI: Five Years of Accomplishments, B. Am. Meteorol. Soc., 93, 347-370, https://doi.org/10.1175/bams-d-11-00027.1, 2012.

Hindley, N. P., Smith, N. D., Wright, C. J., Rees, D. A. S., and Mitchell, N. J.: A two-dimensional Stockwell transform for gravity wave analysis of AIRS measurements, Atmos. Meas. Tech., 9, 2545-2565, https://doi.org/10.5194/amt-9-2545-2016, 2016.

Hindley, N. P., Wright, C. J., Smith, N. D., Hoffmann, L., Holt, L. A., Alexander, M. J., Moffat-Griffin, T., and Mitchell, N. J.: Gravity waves in the winter stratosphere over the Southern Ocean: high-resolution satellite observations and 3D spectral analysis, Atmos. Chem. Phys., 19, 15377-15414, https://doi.org/10.5194/acp-19-15377-2019, 2019.

Hindley, N. P., Wright, C. J., Hoffmann, L., Moffat-Griffin, T., and Mitchell, N. J.: An 18-Year Climatology of Directional Stratospheric Gravity Wave Momentum Flux From 3-D Satellite Observations, Geophys. Res. Lett., 47, e2020GL089557, https://doi.org/10.1029/2020gl089557, 2020.

Hoffmann, L. and Alexander, M. J.: Retrieval of stratospheric temperatures from Atmospheric Infrared Sounder radiance measurements for gravity wave studies, J. Geophys. Res., 114, D07105, https://doi.org/10.1029/2008jd011241, 2009.

Hoffmann, L., Alexander, M. J., Clerbaux, C., Grimsdell, A. W., Meyer, C. I., Rößler, T., and Tournier, B.: Intercomparison of stratospheric gravity wave observations with AIRS and IASI, Atmos. Meas. Tech., 7, 4517-4537, https://doi.org/10.5194/amt-74517-2014, 2014.
Jackson, D. R., Gadian, A., Hindley, N. P., Hoffmann, L., Hughes, J., King, J., Moffat-Griffin, T., Moss, A. C., Ross, A. N., Vosper, S. B., Wright, C. J., and Mitchell, N. J.: The South Georgia Wave Experiment: A Means for Improved Analysis of Gravity Waves and Low-Level Wind Impacts Generated from Mountainous Islands, B. Am. Meteorol. Soc., 99, 1027-1040, https://doi.org/10.1175/bams-d-16-0151.1, 2018.

Liu, X., Xu, J., Yue, J., and Vadas, S. L.: Numerical modeling study of the momentum deposition of small amplitude gravity waves in the thermosphere, Ann. Geophys., 31, 1-14, https://doi.org/10.5194/angeo-31-1-2013, 2013.

Perrett, J. A., Wright, C. J., Hindley, N. P., Hoffmann, L., Mitchell, N. J., Preusse, P., Strube, C., and Eckermann, S. D.: Determining Gravity Wave Sources and Propagation in the Southern Hemisphere by Ray-Tracing AIRS Measurements, Geophys. Res. Lett., 48, e2020GL088621, https://doi.org/10.1029/2020gl088621, 2021.

Stockwell, R., Mansinha, L., and Lowe, R.: Localization of the complex spectrum: the $S$ transform, IEEE T. Signal Proces., 44, 9981001, https://doi.org/10.1109/78.492555, 1996.

Stockwell, R. G.: A basis for efficient representation of the S-transform, Digit. Signal Process., 17, 371-393, https://doi.org/10.1016/j.dsp.2006.04.006, 2007.

Wang, Y. and Orchard, J.: Fast Discrete Orthonormal Stockwell Transform, SIAM J. Sci. Comput., 31, 4000-4012, https://doi.org/10.1137/080737113, 2009.

Wright, C.: Detection of stratospheric gravity waves using HIRDLS data, PhD thesis, University of Oxford, Oxford, UK, 2010.

Wright, C.: corwin365/Phase-Shift-ST: Initial Release as submitted to journal (Version 1), Zenodo [code], https://doi.org/10.5281/zenodo.4569067, 2021.

Wright, C. J. and Gille, J. C.: Detecting overlapping gravity waves using the S-Transform, Geophys. Res. Lett., 40, 1850-1855, https://doi.org/10.1002/grl.50378, 2013.

Wright, C. J., Hindley, N. P., and Mitchell, N. J.: Combining AIRS and MLS observations for three-dimensional gravity wave measurement, Geophys. Res. Lett., 43, 884-893, https://doi.org/10.1002/2015g1067233, 2016.

Wright, C. J., Hindley, N. P., Hoffmann, L., Alexander, M. J., and Mitchell, N. J.: Exploring gravity wave characteristics in 3-D using a novel S-transform technique: AIRS/Aqua measurements over the Southern Andes and Drake Passage, Atmos. Chem. Phys., 17, 8553-8575, https://doi.org/10.5194/acp17-8553-2017, 2017. 